\title{
The probability distribution of Brownian motion in periodic potentials
}

\author{
Matan Sivan ${ }^{1}$ and Oded Farago ${ }^{1,2}$ \\ ${ }^{1}$ Department of Biomedical Engineering, Ben-Gurion University of the Negev, Be'er Sheva 85105, Israel \\ ${ }^{2}$ Ilse Katz Institute for Nanoscale Science and Technology, \\ Ben-Gurion University of the Negev, Be'er Sheva 85105, Israel
}

\begin{abstract}
We calculate the probability distribution function (PDF) of an overdamped Brownian particle moving in a periodic potential energy landscape $U(x)$. The PDF is found by solving the corresponding Smoluchowski diffusion equation. We derive the solution for any periodic even function $U(x)$, and demonstrate that it is asymptotically (at large time $t$ ) correct up to terms decaying faster than $\sim t^{-3 / 2}$. As part of the derivation, we also recover the Lifson-Jackson formula for the effective diffusion coefficient of the dynamics. The derived solution exhibits agreement with Langevin dynamics simulations when (i) the periodic length is much larger than the ballistic length of the dynamics, and (ii) when the potential barrier $\Delta U=\max (U(x))-\min (U(x))$ is not much larger than the thermal energy $k_{B} T$.
\end{abstract}

\section{INTRODUCTION}

Brownian motion in a periodic potential constitutes one of the fundamental problems of particle transport with numerous applications in various fields of science and technology. Many classical examples of diffusion in periodic systems are found in the area of condensed matter physics, including diffusion of atoms in and on the surface of lattices [1, 2], and fluctuations of Josephson supercurrent through a tunneling junction [3]. In many situations, e.g., the cases of superionic conductors [4] and rotating dipoles in external fields [5], a constant force which biases the stochastic dynamics in a given direction is also present. In such scenarios, often referred to as diffusion in a tilted periodic potential different types of dynamics are observed in the overdamped (high friction) regime depending on whether the total potential energy (the sum of periodic and linear potentials) has minima or not [6]. In the former case, the particle moves from one minimum to another and the solution is termed "locked"; in the latter case, the particle moves down the corrugated potential gradient and the solution in termed "running" 7].

The problem of diffusion in periodic systems is also relevant to the study of thermal ratchets [8 10]. Thermal ratchets employ a time-varying spatially-asymmetric periodic potential that drives isothermal systems out of equilibrium and allows for the rectification of the thermal noise in the form of a directed probability (particle) flux. Thermal ratchets attracted much renewed interest in the 1990s' as possible models for motor proteins [11]. Advances in various experimental techniques, most notably in optical trapping ("tweezers") devices, have led to novel experimental setups where some of the new theoretical concepts were tested [12]. Another closely-related problem attracting considerable recent attention is diffusion in corrugated channels [13]. Understanding Brownian motion in confined geometries is important for the study of transport of materials in, e.g., zeolites [14] and microfluidic channels [15]. Such dynamics can be studied by considering a one-dimensional Fick-Jacobs diffusion equation of a particle moving in the presence of an effective potential of mean force arising from the variations in the cross-sectional area of the channel [16, 17].

Here, we consider one-dimensional diffusion in the high friction regime. The probability distribution function (PDF), $P(x, t)$, of finding the particle at coordinate $x$ at time $t$ can be found by solving the Smoluchowski equation [18]

$$
\frac{\partial P(x, t)}{\partial t}=D \frac{\partial}{\partial x}\left\{e^{-\beta U(x)} \frac{\partial}{\partial x}\left[e^{\beta U(x)} P(x, t)\right]\right\}
$$

where $D$ is the diffusion coefficient of the medium, $U(x)$ is the potential energy function, and $\beta=1 / k_{B} T$ where $T$ is the temperature and $k_{B}$ is Boltzmann's constant. Throughout the paper, we assume that $U(x)$ is an even periodic function with period $\lambda$ and consider a particle initially located at the origin, i.e., $P(x, t=0)=\delta(x)$, where $\delta$ is the Dirac delta-function. From symmetry considerations, the mean displacement of the particle vanishes identically, $\langle x\rangle=0$. The mean-squared displacement does not vanish but rather exhibits, at asymptotically large times, a linear growth with time characterizing the diffusive nature of the dynamics. However, the effective diffusion coefficient defined by

$$
D^{*}=\lim _{t \rightarrow \infty} \frac{\left\langle x^{2}\right\rangle}{2 t}
$$

is not equal to the medium diffusion coefficient, but is given by the Lifson-Jackson (LJ) formula [19]

$$
D^{*}=\frac{D}{\left\langle e^{-\beta U(x)}\right\rangle\left\langle e^{\beta U(x)}\right\rangle},
$$

where $\langle\cdots\rangle$ denotes an average over a unit cell: $\langle c\rangle=$ $(\lambda)^{-1} \int_{0}^{\lambda} c(x) d x$. This formula has been derived by several authors using somewhat different approaches 20 22], and it has been proved that $D^{*} \leq D[20]$. Physically, the fact that $D^{*} \leq D$ is directly related to the tendency of the Brownian particle to get trapped, for some duration, in the minima of the periodic potential before moving to the adjacent cell. 
We note here that the LJ formula is valid only when the potential barrier, $\Delta U=U_{\max }-U_{\min }$ is not too high. In the high barrier limit, $\beta \Delta U \gg 1$, the particle only rarely escapes the vicinity of a potential minimum, and it advances to a neighboring cell with a characteristic time that scales as $\tau \sim \exp (\beta \Delta U)$. In this limit, the effective diffusion coefficient is expected to follow an ArrheniusKramer behavior [23]: $D^{*} \sim D e^{-\beta \Delta U}$.

Despite the extensive theoretical literature on the problem of diffusion in periodic potentials, the general solution of Eq. (1) subject to the delta-function initial condition is not known. Here, we take a major step forward and derive an asymptotic (at large times) solution for the Smoluchowski equation for any even periodic potential function $U(x)$. We use Langevin dynamics simulations of a case-study to demonstrate excellent agreement between our analytical solution and the computed PDF. As part of our derivation, we independently arrive at the LJ formula for the effective diffusion coefficient.

\section{PRELIMINARY CONSIDERATIONS}

Although we focus here on diffusive (overdamped) dynamics, our computational results are actually based on inertial (underdamped) Langevin dynamics simulations. The Langevin equation is given by 24]

$$
m \frac{d v}{d t}=-\alpha v+\xi(t)+f(x)
$$

where $m$ and $v$ denote, respectively, the mass and velocity of the particle, $f=-d U(x) / d x$ is the deterministic force acting on the particle, $\alpha>0$ is the medium's friction coefficient, and $\xi(t)$ is a thermal white nosie with zero average $\langle\xi(t)\rangle=0$ and delta function auto-correlation $\left\langle\xi(t) \xi\left(t^{\prime}\right)\right\rangle=2 k_{B} T \alpha \delta\left(t-t^{\prime}\right)$ [7]. Langevin dynamics is inertial at time scales much smaller than the ballistic time $\tau_{b} \sim m / \alpha$, during which the particle moves a characteristic distance $l_{b} \sim v_{\mathrm{th}} \tau_{b}$, where $v_{\mathrm{th}}=\sqrt{k_{B} T / m}$ is the thermal velocity of the particle. The dynamics becomes diffusive at length scales much larger than $l_{b}$. From Smoluchowski equation (1) with $D=k_{B} T / \alpha$ (Einstein's relation), one can derive the particle's PDF in the overdamped limit of Langevin's equation, i.e., when $l_{b} \rightarrow 0$. This description, however, is valid only if the spatial variations of the deterministic force on length scales of the order of $l_{b}$ are much smaller than the characteristic friction force, i.e., for $\left(m / \alpha^{2}\right)|d f / d x| \ll 1$ [25]. In the case of a periodic force $|d f / d x| \sim \Delta U / \lambda^{2}$, implying that Eq. (1) may not be valid when the periodic length of the potential becomes comparable to the ballistic length, or in the case when $\Delta U \gg k_{B} T$. We will later see that, indeed, in these limits, the LJ formula derived from Eq. (1) fails to depict correctly the effective diffusion coefficient $D^{*}$.

We notice that if $U(x)$ is periodic then the Boltzmann's weight $\exp [-\beta U(x)]$ is also a periodic function. We thus define the periodic function $\eta(x)$

$$
1+\epsilon \eta(x)=\frac{e^{-\beta U(x)}}{\left\langle e^{-\beta U(x)}\right\rangle},
$$

with the variable

$$
\epsilon=1-\frac{e^{-\beta U_{\max }}}{\left\langle e^{-\beta U(x)}\right\rangle}
$$

satisfying $0 \leq \epsilon<1$. The function $\eta(x)$ has the following properties: (i) $\langle\eta(x)\rangle=0$, and (ii) $\min [\eta(x)]=-1$. With the definition of $\epsilon$ and $\eta(x)$, the Smoluchowski equation (1) takes the form

$$
\frac{\partial P(x, t)}{\partial t}=D \frac{\partial}{\partial x}\left\{[1+\epsilon \eta(x)] \frac{\partial}{\partial x} \frac{P(x, t)}{[1+\epsilon \eta(x)]}\right\},
$$

and LJ formula (3) reads

$$
D^{*}=\frac{D}{\left\langle[1+\epsilon \eta(x)]^{-1}\right\rangle} .
$$

In the high barrier limit $\epsilon \rightarrow 1$, we expect the ArrheniusKramer law which takes the form

$$
D^{*} \sim D(1-\epsilon) .
$$

\section{LANGEVIN DYNAMICS SIMULATIONS}

We simulate the dynamics of a particle of unity mass $m=1$ moving in a system with friction coefficient $\alpha=1$ at constant temperature $k_{B} T=1$. For this choice of parameters the ballistic time $\tau_{b} \sim m / \alpha=1$ and ballistic length $l_{b} \sim \sqrt{m k_{B} T} / \alpha=1$. As a numerical example, we consider the case where $\eta(x)=\cos (2 \pi x / \lambda)$ $\left(f(x)=-d U(x) / d x=k_{B} T \epsilon \eta^{\prime}(x) /[1+\epsilon \eta(x)]\right)$. The particle's trajectory begins at $x=0$ with initial velocity which is drawn from the equilibrium MaxwellBoltzmann distribution, and is numerically integrated using the algorithm of Grønbech-Jensen and Farago (GJF) [26, 27] with $d t=0.1$, which is an order of magnitude smaller than $\tau_{b}$. The numerical results presented here are based on statistical averages of $2 \times 10^{8}$ independent trajectories. Fig. 1(a) shows the ratio between $\left\langle x^{2}\right\rangle$ and $2 t$ [see Eq. (2)] as a function of $t$ for $\epsilon=0.5$ and $\lambda=50 \gg l_{b}$. At asymptotically large times, this ratio converges to the effective diffusion coefficient $D^{*} \simeq 0.863$ which is indeed smaller than the medium diffusion coefficient $D=k_{B} T / \alpha=1$. The open circles in Fig. 1)(b) show our computational results for $D^{*} / D$ as a function of $\epsilon$, for $\lambda=50$. The results exhibit excellent agreement with the solid line depicting the LJ formula (8) which, for the specific choice of $\eta(x)$ discussed here, gives $D^{*} / D=\sqrt{1-\epsilon^{2}}$. Very small deviations from the LJ formula are observed for $\epsilon>0.6$ when the potential barrier becomes larger $[\exp (-\beta \Delta U) \lesssim 0.17]$. In contrast, the results for $\lambda=2$, 

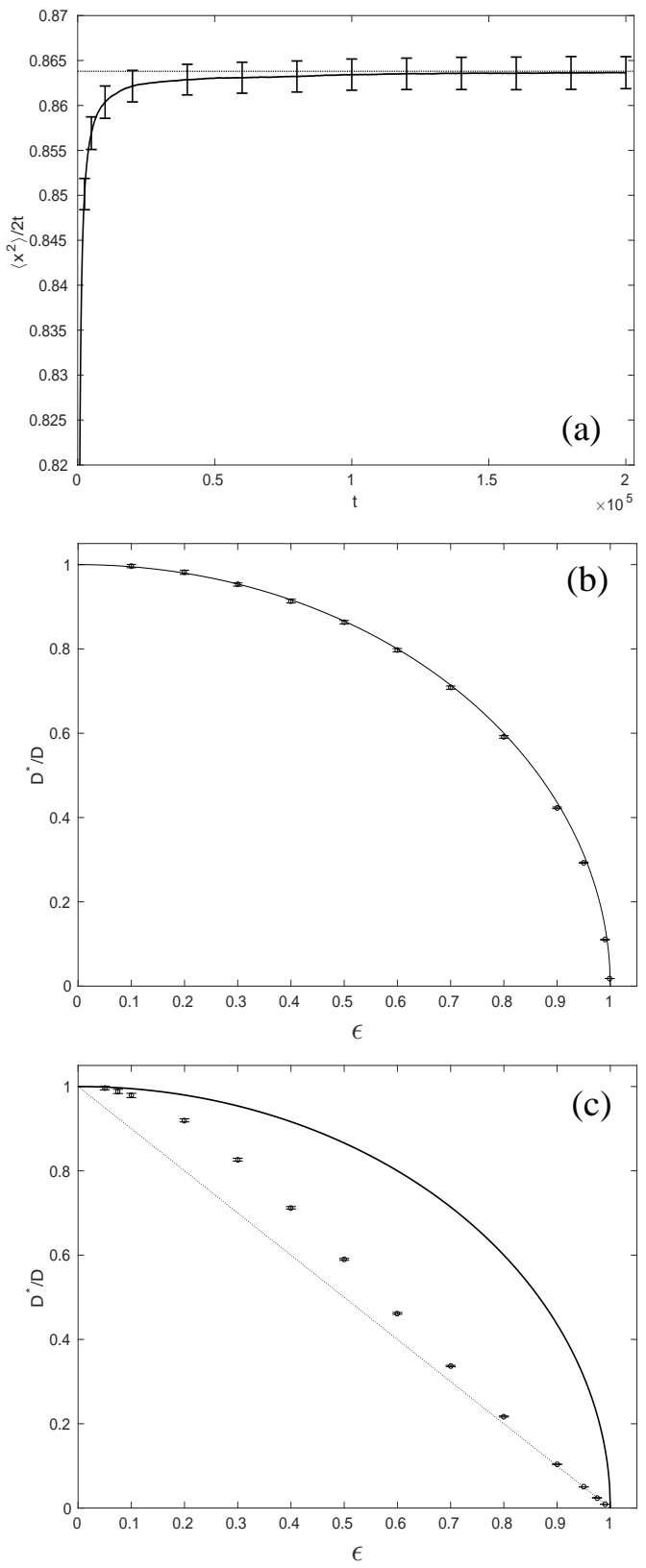

FIG. 1: (a) The ratio between the mean squared displacement and twice the time, $D^{*}=\left\langle x^{2}\right\rangle / 2 t$, as a function of $t$ for $\epsilon=0.5$ and $\lambda=50$. At large times this ratio converges to $D^{*} \simeq 0.863$ that matches the value for the effective diffusion coefficient $D^{*}$ predicted by the LJ formula. (b) The effective diffusion coefficient $D^{*}$ (normalized by the medium diffusion coefficient $D$ ) as a function of $\epsilon$ for $\lambda=50$. Circles - simulation results, solid curve - the LJ formula. (c) Same as (b) for $\lambda=2$. The dashed line depicts the function $(1-\epsilon)$.

which are plotted in Fig. 1(c), exhibit agreement with LJ formula only for $\epsilon<0.1$. This behavior is expected since LJ formula is derived from the Smoluchowski equation, but the latter becomes invalid when $\lambda$ is comparable to the ballistic length $l_{b}$. For $\epsilon \rightarrow 1$, we observe that $D^{*} / D$ diminishes like $(1-\epsilon)$, in accordance with Eq. (9).
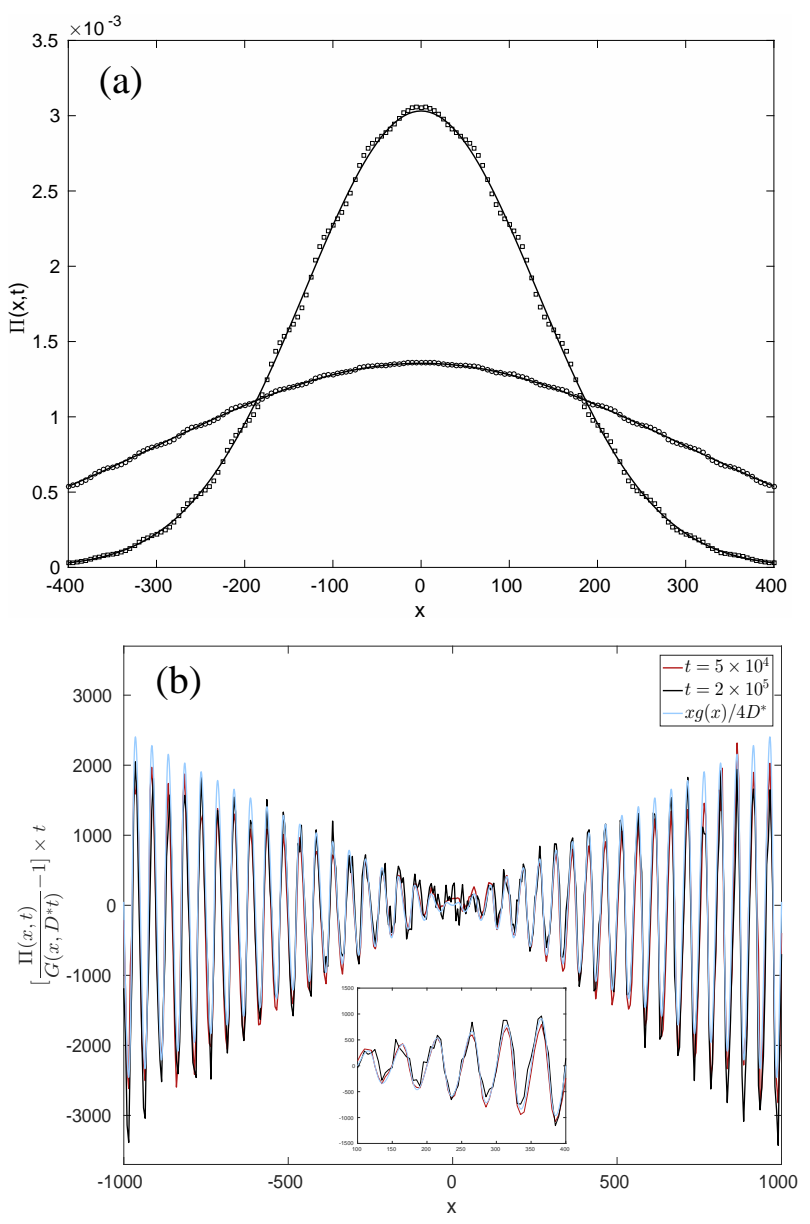

FIG. 2: (Color Online) (a) The function $\Pi(x, t)$ as a function of $x$ for $\epsilon=0.5$ and $\lambda=50$, at $t=10^{4}$ (squares) and and $t=5 \times 10^{4}$ (circles). The solid curves depict the Gaussian form $G\left(x, D^{*} t\right)=\exp \left(-x^{2} / 4 D^{*} t\right) / \sqrt{4 \pi D^{*} t}$ with $D^{*}=0.863$. (b) The function $t\left[\Pi(x, t) / G\left(x, D^{*} t\right)-1\right]$ as a function of $x$ for $t=5 \times 10^{4}$ (red) and $t=2 \times 10^{5}$ (black). The light blue curve is the analytical approximation $x g(x) /\left(4 D^{*}\right)$ with $g(x)$ given by Eq. (18). The inset shows a magnification of the region with the best agreement between the analytical approximation and numerical results.

Fig. 2(a) shows the PDF normalized by the Boltzmann factor, $\Pi(x, t)=P(x, t) /[1+\epsilon \eta(x)]$, for $\epsilon=0.5$ and $\lambda=50$ at $t=10^{4}$ (squares) and $t=5 \times 10^{4}$ (circles). The graphs indicate that at large times, the function $\Pi(x, t)$ is very well approximated by a Gaussian form (solid lines) $G\left(x, D^{*} t\right)=\exp \left(-x^{2} / 4 D^{*} t\right) / \sqrt{4 \pi D^{*} t}$, where $D^{*}=0.863$ is the effective diffusion coefficient for the corresponding values of $\epsilon$ and $\lambda$ (see above). This, however, is only an approximation, and it is straightforward to check that $P(x, t)=[1+\epsilon \eta(x)] G\left(x, D^{*} t\right)$ is not a solution of Eq. (7). Careful inspection of Fig. 2(a) reveals small undulations of $\Pi(x, t)$ with wavelength $\lambda$ around the Gaussian form. We thus speculate that the Gaussian form is the leading term in an expression including additional terms, and write $P(x, t)=$ 
$[1+\epsilon \eta(x)] G\left(x, D^{*} t\right)\left[1+q_{0}(x)+q_{1}(x, t)\right]$, where $q_{0}(x)$ and $q_{1}(x, t)$ denote, respectively, static and time-decaying corrections exhibiting oscillations with periodicity $\lambda$. The static term can be ruled out based on the simple argument that it contributes a term equal to $G\left(x, D^{*} t\right) d\{[1+$ $\left.\epsilon \eta(x)] q_{0}^{\prime}(x)\right\} / d x$ on the right hand side of Eq. (7), and this term dominates the asymptotic behavior of $\partial_{t} P(x, t)$ at long times. Since the Gaussian function $G\left(x, D^{*} t\right)$ is positive, it follows that the long time limit of $\partial_{t} P(x, t)$ has the same sign as the static function $d\left\{[1+\epsilon \eta(x)] q_{0}^{\prime}(x)\right\} / d x$ that oscillates between positive and negative values [28]. This, however, is impossible because the particle propagates to further distances with time and the probability density cannot accumulate at any point in space. Stated differently, for any $x_{0}$, the probability density $P\left(x_{0}, t\right)$ must decay at large times, namely $\partial_{t} P\left(x_{0}, t\right)<0$ for some $t>t_{0}$. This property of $P(x, t)$ precludes the possibility of a static correction $q_{0}(x)$ and allows only a timedecaying term $q_{1}(x, t)$.
Insight into the form of the time-decaying correction can be gained from Fig. 2(b) where we plot the function $t\left[\Pi(x, t) / G\left(x, D^{*} t\right)-1\right]=t q_{2}(x, t)$ as a function of $x$ for $\epsilon=0.5$ and $\lambda=50$, at $t=5 \times 10^{4}$ (red) and $t=2 \times 10^{5}$ (black). While the collapse of the data for the different times in not perfect, it seems to indicate that the time-decaying correction may have the form $q_{1}(x, t) \sim(x / t) g(x)$, where $g(x)$ is a scaling function with periodicity $\lambda$. This term decays at a rate $t^{1 / 2}$ faster than the leading Gaussian form because of the scaling $x \sim\left(D^{*} t\right)^{1 / 2}$. (Larger values of $x$ need not be considered because for $x^{2} \gg D^{*} t$ the PDF is practically zero.) The solution can be further refined by introducing a series of corrections $P(x, t)=[1+$ $\epsilon \eta(x)] G\left(x, D^{*} t\right)\left[1+q_{1}(x, t)+q_{2}(x, t)+\cdots\right]$, with each term decaying $t^{1 / 2}$ faster than the previous one. Here, we attempt to find the first two leading corrections and, therefore, consider the following form

$$
P(x, t)=\left\{[1+\epsilon \eta(x)] G\left(x, D^{*} t\right)\left[1+\frac{\lambda x g(x)}{4 D^{*} t} Q_{1}\left(\frac{x^{2}}{4 D^{*} t}\right)+\frac{\lambda^{2} h(x)}{4 D^{*} t} Q_{2}\left(\frac{x^{2}}{4 D^{*} t}\right)\right]\right\}
$$

where $g(x)$ and $h(x)$ are two dimensionless functions with periodicity $\lambda$, while $Q_{1}$ and $Q_{2}$ are polynomials in $x^{2} / 4 D^{*} t$. This solution constitutes the asymptotic solution to Smoluchowski equation (7) at large times $t \gg \tau_{b}$, up to order $G\left(x, D^{*} t\right) / t \sim 1 / t^{3 / 2}$.

\section{THE PROBABILITY DISTRIBUTION}

\section{A. The leading asymptotic correction}

We consider a symmetric system with an even function $\eta(x)$, which implies that the PDF is symmetric and, therefore, $g(x)$ must be an odd function while $h(x)$ is even. Both scaling functions are periodic with periodicity $\lambda$ and have a finite amplitude. Thus, $\eta^{\prime}(x)$, as well as $g^{\prime}(x)$ and $h^{\prime}(x)$, are all of order $1 / \lambda$. With this in mind, we substitute the solution (10) into Eq. (7). On the right hand side, we find terms that scale as like $G\left(x, D^{*} t\right)(x / \lambda t)$. These terms must cancel each other, which occurs provided that (i) $Q_{1}=$ Const (which, by a proper definition of $g(x)$, can be arbitrarily set to unity), and (ii) the scaling function $g(x)$ satisfies the ordinary differential equation

$$
[1+\epsilon \eta(x)] \lambda g^{\prime \prime}(x)+\epsilon \eta^{\prime}(x)\left[\lambda g^{\prime}(x)-2\right]=0 .
$$

Taking into account that $g(x)$ is an odd function and therefore $g(0)=0$, we readily arrive at the solution

$$
\lambda g(x)=k I\left[\frac{1}{1+\epsilon \eta(x)}\right]+2 x
$$

where $I[y(x)]$ denotes the primitive function of $y(x)$ with $I(x=0)=0$. The constant $k$ can be found from the requirement that $g(x)$ is periodic. Thus, $\int_{0}^{\lambda} g^{\prime}(x) d x=$ $g(\lambda)-g(0)=0$, which gives

$$
k=-\frac{2}{\left\langle[1+\epsilon \eta(x)]^{-1}\right\rangle} .
$$

\section{B. The next asymptotic correction}

We now proceed and find the function $h(x)$ and the polynomial $Q_{2}$ by comparing terms of the form $G\left(x, D^{*} t\right)\left(x^{2} / 4 D^{*} t\right)^{n} / t$ (where $n$ is an integer), on both sides of Eq. (77). From this comparison, we readily conclude that $Q_{2}\left(x^{2} / 4 D^{*} t\right)=\left[1+b x^{2} /\left(4 D^{*} t\right)\right]$. This leaves us with two different differential equations for the scaling function $h(x)$ which cannot be solved simultaneously unless we set $b=-2$, in which case the equations coincide and read

$$
\begin{aligned}
& (1+\phi)[1+\epsilon \eta(x)]\left[\lambda^{2} h^{\prime \prime}(x)+2 \lambda g^{\prime}(x)\right]+ \\
& (1+\phi) \epsilon \eta^{\prime}(x)\left[\lambda^{2} h^{\prime}(x)+\lambda g(x)\right]=2 \phi([1+\epsilon \eta(x)],
\end{aligned}
$$

where $\phi=D / D^{*}-1$. By defining the function $\tilde{h}(x)=$ $[1+\epsilon \eta(x)]\left[\lambda^{2} h^{\prime}(x)+\lambda g(x)\right]$, we arrive at the simple equation

$$
\tilde{h}^{\prime}(x)=-\left[\frac{2}{1+\phi}+k\right]-\frac{2 \epsilon \eta(x)}{1+\phi},
$$


which can be integrated once to yield $\tilde{h}(x)$, from which the scaling function $h(x)$ can be derived by performing yet another integration in $x$. Importantly, the fact that $h(x)$ is an even periodic function with a finite amplitude imposes the relationship $2 /(1+\phi)+k=0$ from which $D^{*}$ can be deduced. Using Eq. (13) we find

$$
D^{*}=\frac{D}{1+\phi}=-\frac{k D}{2}=\frac{D}{\left\langle[1+\epsilon \eta(x)]^{-1}\right\rangle}
$$

which is identical to the LJ formula (8). The scaling function $h(x)$ is given by

$$
\lambda^{2} h(x)=k I\left\{\frac{I[\epsilon \eta(x)]}{1+\epsilon \eta(x)}-I\left[\frac{1}{1+\epsilon \eta(k)}\right]\right\}-x^{2}+\lambda^{2} C,
$$

where the constant $C$ is determined by the normalization condition $\int_{-\infty}^{\infty} P(x, t) d x=1$ (which must be satisfied up to an order of $1 / t)$.

\section{Comparison with simulation results}

Returning to our simulation results above for $\eta(x)=$ $\cos (2 \pi x / \lambda)$, the corresponding scaling function $g(x)$ can be found

$$
g(x)=-\frac{2}{\pi} \arctan \left[\sqrt{\frac{1-\epsilon}{1+\epsilon}} \tan \left(\frac{\pi x}{\lambda}\right)\right]+\frac{2 x}{\lambda},
$$

where the arctan function is interpreted such that it returns a value between $-\pi / 2$ and $\pi / 2$ which is then shifted by an integer number of $\pi$ in order that $g(\lambda / 2+n \lambda)=0$ (where $n$ is an integer). We were unable to analytically perform the integration in Eq. (17), necessary for finding a closed-form expression for the scaling function $h(x)$; however, we take advantage of the fact that it represents a correction to the Gaussian form of $\Pi(x, t)$ which is asymptotically smaller than the one involving the function $g(x)$ [see Eq. (10)], and use the approximation $t\left[\Pi(x, t) / G\left(x, D^{*} t\right)-1\right] \simeq x g(x) /\left(4 D^{*}\right)$. This approximation, which is plotted in Fig. 2(b) in light blue, shows a good fit to the simulation results. The deviations, which can be attributed to the higher order correction term in Eq. (10), are particularly small for $x^{2} \simeq 2 D^{*} t$ - see inset in Fig. 2(b).

\section{SUMMARY AND FUTURE OUTLOOK}

In summary, we derived an asymptotic (at large times) expression for the PDF of a particle diffusing in a periodic potential energy landscape $U(x)$. The solution, which is given by Eq. (10), is correct to order $1 / t^{3 / 2}$. Faster decaying corrections can, in principle, be systematically derived by comparing, on both sides of Eq. (77), terms of order $1 / t^{2}, 1 / t^{5 / 2} \ldots$ This will require solving increasingly complicated differential equations involving the scaling functions of slower decaying terms.

We conclude by noting that our approach to solving the Smoluchowski equation can be used, with only simple modifications, to solving other closely-related diffusion equation. These include, for instance, the equation describing Brownian motion in a tilted periodic potential, i.e., when a particle diffuses under the action of both a periodic potential $U(x)$ and a constant force $f$. In this case, the Smoluchowski equation reads

$$
\begin{aligned}
& \frac{\partial P(x, t)}{\partial t}=D \frac{\partial}{\partial x}\left\{e^{-\beta(U(x)-f x)} \frac{\partial}{\partial x}\left[e^{\beta(U(x)-f x)} P(x, t)\right]\right\} \\
& =-\frac{f}{\alpha} \frac{\partial P}{\partial x}+D \frac{\partial}{\partial x}\left\{[1+\epsilon \eta(x)] \frac{\partial}{\partial x} \frac{P(x, t)}{[1+\epsilon \eta(x)]}\right\},
\end{aligned}
$$

where $\alpha=k_{B} T / D$, while $\eta(x)$ and $\epsilon$ are defined similarly to Eqs. (5) and (6). The two main differences with respect to the case $f=0$ considered previously: (i) The Gaussian "moves" at constant velocity $f / \alpha^{*}$, where $\alpha^{*} \neq \alpha$ is the effective friction coefficient satisfying $\alpha^{*} \rightarrow k_{B} T / D^{*}$ for $f \rightarrow 0$ [21]. (ii) Corrections to the Gaussian form also include a static term $q_{0}(x)$ missing when $f=0$, with oscillations having the wavelength $\lambda$ of the potential $U(x)$. Thus, we speculate that the solution takes the form

$$
\begin{aligned}
P(x, t) & =[1+\epsilon \eta(x)] G\left(x-\frac{f}{\alpha^{*}} t, D^{*} t\right) \times \\
& \times\left[1+q_{0}(x)+q_{1}(x, t)+q_{2}(x, t)+\cdots\right],
\end{aligned}
$$

where $G=\exp \left[-\left(x-t f / \alpha^{*}\right)^{2} / 4 D^{*} t\right] / \sqrt{4 \pi D^{*} t}$ denotes the "running" Gaussian, while $q_{1}$ and $q_{2}$ are the time decaying corrections of order $1 / t^{1 / 2}$ and $1 / t$, respectively, having the same general form as in Eq. (10) of the main text.

Upon substituting the solution (20) into Eq. (19) and comparing terms of similar order, we first arrive at the following differential equation for the static term $q_{0}(x)$

$$
\frac{\partial}{\partial x}\left\{[1+\epsilon \eta(x)]\left[\beta f+\beta f q_{0}(x)-q_{0}^{\prime}(x)\right]\right\}=0 .
$$

From the requirements that (i) $q_{0}(x)$ does not diverge exponentially for $x \rightarrow \infty$, and that (ii) $P(x, t)$ is normalized to unity at any time (including for $t \rightarrow \infty$, when the time-decaying terms become irrelevant), we arrive at

$$
q_{0}(x)=\beta f e^{\beta f x} I\left[e^{-\beta f x}\left(\frac{c_{1}}{1+\epsilon \eta(x)}+1\right)\right],
$$

where (as above) $I$ denote a primitive function, and

$$
c_{1}=\left\{\beta f\left\langle[1+\epsilon \eta(x)] e^{\beta f x} I\left[\frac{e^{-\beta f x}}{1+\epsilon \eta(x)}\right]\right\rangle\right\}^{-1} .
$$

The first time-decaying correction $q_{1}(x)$ can be now found by solving the equation obtained from the terms that scale as $1 / t^{1 / 2}$. This equation involves the already 
found static scaling function $q_{0}(x)$. Then, the second correction, $q_{2}(x)$, can be found from the equation corresponding to the terms proportional to $1 / t$, which involves $q_{0}(x)$ and $q_{1}(x)$ (and so on). Notice that the form of $q_{0}$ (22) does not give any information on the effective param- eters $\alpha^{*}$ and $D^{*}$. These will be found from the equations for $q_{1}$ and $q_{2}$, respectively.

Acknowledgments: This work was supported by the Israel Science Foundation (ISF) through grant No. 991/17.
[1] J. H. Weiner and R. E. Froman, Phys. Rev. B 10, 315 (1974).

[2] T. Ala-Nissila, R. Ferrando, and S. C. Ying, Adv. Phys. 51, 949 (2002).

[3] V. Ambegaokar and B. I. Halperin, Phys. Rev. Lett. 22, 1364 (1969).

[4] P. Fulde, L. Pietronero, W. R. Schneider, and S. Strässler, Phys. Rev. Lett. 35, 1776 (1975).

[5] D. Reguera, J. M. Rubi, and A. Pérez-Madrid, Phys. Rev. E 62, 5313 (2000).

[6] P. Reimann, C. Van den Broeck, H. Linke, P. Hänggi, J. M. Rubi, and A. Pérez-Madrid, Phys. Rev. E 65, 031104 (2002).

[7] H. Risken, The Fokker-Planck Equation (Springer, Berlin, 1984).

[8] P. Reimann, Phys. Rep. 361, 57 (2002).

[9] P. Hnggi and F. Marchesoni, Rev. Mod. Phys. 81, 387 (2009).

[10] D. Cubero and F. Renzoni, Brownian Ratchets: From Statistical Physics to Bio and Nano-motors, (Cambridge University Press, 2016).

[11] M. Bier, Contemp. Phys. 38, 371 (1997).

[12] B. A. Koss and D. G. Grier, Appl. Phys. Lett. 82, 3985 (2003).

[13] R. Zwanzig, J. Phys. Chem. 96. 3926 (1992).

[14] A. Schüring, S. M. Auerbach, S. Fritzsche, and R. Haberlandt, J. Chem. Phys. 116, 10890 (2002).
[15] B. H. Weigl and P. Yager, Science 283, 346 (1999).

[16] D. Reguera and J. M. Rubi, Phys. Rev. E 64, 061106 (2001).

[17] P. Kalinay and J. K. Percus, Phys. Rev. E 74, 041203 (2006).

[18] M. v. Smoluchowski, Ann. Physik 48, 1103 (1915).

[19] S. Lifson and J. L. Jackson, J. Chem. Phys. 36, 2410 (1962).

[20] R. Festa and E. Galleani d'Agliano, Physica A 90, 229 (1978).

[21] L. Gunther, Physica A 95, 367 (1979).

[22] D. L. Weaver, Physica A 98, 359 (1979).

[23] R. Ferrando, R. Spadacini, and G.E. Tommei, Surf. Sci. 265, 273 (1992).

[24] P. Langevin, C. R. Acad. Sci. (Paris) 146, 530 (1908).

[25] J. Wilemski, J. Stat. Phys. 14, 153 (1976).

[26] N. Grønbech-Jensen, and O. Farago. Mol. Phys. 111, 983 (2013).

[27] N. Grønbech-Jensen, N. R. Hayre, and O. Farago, Comput. Phys. Commun. 185, 524 (2014).

[28] The last statement is incorrect when $q_{0}^{\prime}(x)=[1+$ $\epsilon \eta(x)]^{-1}$. However, this possibility can be ruled out since in this case the average slope of $q_{0}$ does not vanish, which implies that $q_{0}(x)$ and, therefore, also the probability density $P(x, t)$ obtain large negative values for some $x$. 